\title{
Radiation-associated colon cancer: A case report
}

\author{
KAZUHITO SASAKI ${ }^{1}$, SOICHIRO ISHIHARA ${ }^{1}$, KEISUKE HATA ${ }^{1}$, \\ TOMOMICHI KIYOMATSU ${ }^{1}$, HIROAKI NOZAWA ${ }^{1}$, KAZUSHIGE KAWAI ${ }^{1}$, TOSHIAKI TANAKA ${ }^{1}$, \\ TAKESHI NISHIKAWA ${ }^{1}$, KENSUKE OTANI ${ }^{1}$, KOJI YASUDA ${ }^{1}$, MANABU KANEKO ${ }^{1}$, \\ KOJI MURONO ${ }^{1}$, HIROYUKI ABE ${ }^{2}$, TEPPEI MORIKAWA ${ }^{2}$ and TOSHIAKI WATANABE ${ }^{1}$ \\ ${ }^{1}$ Department of Surgical Oncology, Faculty of Medicine, The University of Tokyo; ${ }^{2}$ Department \\ of Pathology, Graduate School of Medicine, The University of Tokyo, Tokyo 113-8655, Japan
}

Received January 26, 2017; Accepted February 28, 2017

DOI: $10.3892 /$ mco.2017.1252

\begin{abstract}
Radiation-associated colon cancer is a rare clinical entity. We herein describe the case of a patient with radiation-associated colon cancer who had undergone low anterior resection for rectal cancer following preoperative radiotherapy. Certain characteristics of radiation-associated colon cancer are highlighted. The patient was a 48 -year-old man who had undergone low anterior resection for rectal cancer following preoperative radiotherapy at a total dose of $50 \mathrm{~Gy}$, at the age of 29 years. When the patient presented at the University of Tokyo Hospital, 19 years after the surgery, he complained of severe anal pain and frequent defecation. Colonoscopy revealed two flat tumors in the sigmoid colon, located $10 \mathrm{~cm}$ to the oral side of the anastomosis site, which were diagnosed as well-differentiated adenocarcinomas. In addition, colonoscopy identified five flat polyps near the tumors, which were resected endoscopically. Computed tomography and magnetic resonance imaging revealed a mass in the sigmoid colon and no evidence of distant metastasis. Laparoscopic-assisted intersphincteric resection of the rectum and sigmoid colon with diverting ileostomy was performed. There were no specific postoperative complications and the patient was discharged from the hospital on the 20th postoperative day. On pathological examination, the resected rectum and sigmoid colon contained two separate tumors and six flat polyps. The two tumors were diagnosed as well-differentiated adenocarcinomas with invasion of the subserosa and submucosa, respectively. A total of 17 regional lymph nodes without metastasis were resected. The six flat polyps were diagnosed as tubular adenomas. We herein present a case of a radiation-associated colon cancer in a patient who had undergone low anterior resection for
\end{abstract}

Correspondence to: $\operatorname{Dr}$ Kazuhito Sasaki, Department of Surgical Oncology, Faculty of Medicine, The University of Tokyo, 7-3-1 Hongo, Bunkyo-ku, Tokyo 113-8655, Japan

E-mail: sasakik-tky@umin.ac.jp

Key words: rectal cancer, chemoradiotherapy, radiation-associated, colorectal cancer, radiation proctocolitis rectal cancer following preoperative radiotherapy 19 years prior. Colonoscopic surveillance of radiation-associated colon cancer may be indicated for rectal cancer patients treated with preoperative radiotherapy, particularly for those with long-standing radiation-induced colitis.

\section{Introduction}

Radiotherapy plays an important role in the treatment of several patients with pelvic malignancies, such as anorectal, urological and gynecological cancers (1). Approximately $70 \%$ of cancer patients undergo radiotherapy for these malignancies to prolong disease-free survival and reduce local recurrence $(2,3)$. However, radiotherapy may also lead to significant injury of the normal tissues in the radiation field. The gastrointestinal tract is a radiosensitive organ in the pelvis and often proves to be the major limiting factor when determining tolerance to radiation therapy (4).

One of the long-term complications of radiotherapy for pelvic malignancies is the development of large bowel cancer $(3,5)$. Although the exact incidence of this complication and the mechanisms of tumorigenesis have not been fully elucidated, a close association between radiation and the development of colorectal cancer (CRC) has been demonstrated $(6,7)$. Post-irradiation cancer is currently termed radiation-associated cancer, as other predisposing factors, such as lymphedema, adjuvant chemotherapy and genetic abnormalities may be involved (8).

As most radiation treatments are directed to the pelvis, the rectum is at particular risk of injury. The sigmoid colon is also a common site of involvement due to its fixed position at the pelvic brim, and is likely to receive a higher dose of radiation (9). The occurrence of colon and anorectal cancer following pelvic irradiation has been reported, and the majority of the cases were encountered among women who had undergone previous radiation therapy for gynecological cancers, such as uterine cervical cancer $(5,6,8,10)$. Although preoperative chemoradiotherapy (CRT) is currently the standard therapy for locally advanced rectal cancer (11), little attention has been paid to the occurrence of colon cancer following CRT for rectal cancer. We herein describe a case of suspected radiation-associated sigmoid colon cancer following radiotherapy for locally advanced rectal cancer. 


\section{Case report}

A 48-year-old man presented at the University of Tokyo Hospital with severe anal pain and frequent defecation. The patient had undergone low anterior resection for rectal cancer following preoperative radiotherapy at a total dose of $50 \mathrm{~Gy}$ at the age of 29 years. Six years after surgery, contrast-enhanced computed tomography (CT) revealed a fistula between the sigmoid colon and presacral tissue, which subsequently recovered following conservative management. The patient had been followed up regularly without evidence of recurrence for 7 years, after which time he was lost to follow-up. On admission, the results of the general physical examination were normal. On colonoscopic examination, two flat tumors of the sigmoid colon were identified at a distance of $14 \mathrm{~cm}$ from the anal margin, whereas the anastomosis site was located at a distance of $4 \mathrm{~cm}$ from the anal margin (Fig. 1). Biopsies from both lesions revealed well-differentiated adenocarcinomas. In addition, colonoscopy identified five flat polyps near the tumor site; these flat polyps were resected endoscopically and were diagnosed as tubular adenomas. Colonoscopic findings of radiation proctocolitis were not observed. A water-soluble contrast enema revealed an irregular stricture of the sigmoid colon extending from the tumor to the anastomosis (Fig. 2). $\mathrm{CT}$ and magnetic resonance imaging revealed a mass in the sigmoid colon with no evidence of distant metastasis (Fig. 3). Laparoscopic-assisted intersphincteric resection of the rectum and the sigmoid colon with diverting ileostomy was performed. There were no specific complications postoperatively and the patient was discharged from the hospital on the 20th postoperative day.

On pathological examination, the resected rectum and sigmoid colon contained two separate tumors and six flat polyps (Fig. 4A and B). The first tumor was located at $8 \mathrm{~cm}$ from the distal margin and measured $3.5 \times 1.5 \mathrm{~cm}$; the tumor was pathologically diagnosed as well-differentiated adenocarcinoma with invasion of the subserosa (Fig. 4C). Another tumor was located near the first and measured $2.5 \times 2.2 \mathrm{~cm}$; this tumor was pathologically diagnosed as well-differentiated adenocarcinoma with invasion of the submucosa. In addition, 17 regional lymph nodes without metastasis were resected. Microsatellite instability (MSI) was not observed on genetic analysis; however, p53 overexpression was detected using immunohistochemical examination of the first tumor (Fig. 4D). All six flat polyps were diagnosed as tubular adenomas. Pathological findings of radiation proctocolitis, such as submucosal fibrosis and hyalinization of the blood vessels, were not observed in the sigmoid colon surrounding the tumor.

\section{Discussion}

The clinicopathological characteristics of radiation-associated CRC are as follows: i) Prior history of radiation therapy and development of a malignancy in the radiation field; ii) clinically chronic radiation proctocolitis at the site of the subsequent tumor; iii) a long interval of $\geq 10$ years between irradiation and the appearance of the malignancy; iv) histopathological evidence of intestinal radiation damage; and v) mucinous carcinoma as the most common type (5). In the present case, all these characteristics, apart from histological findings of

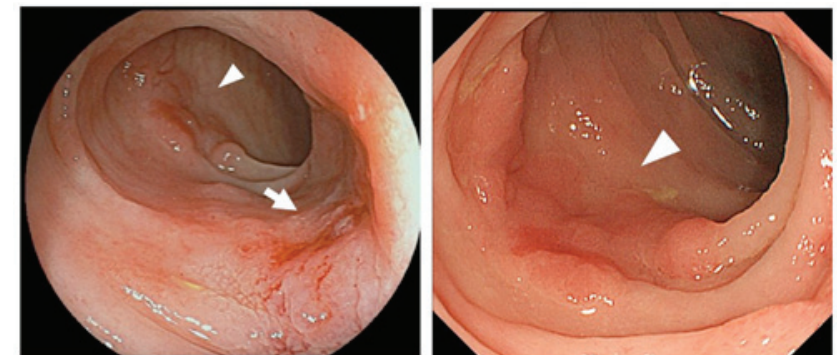

Figure 1. Colonoscopy revealed two flat tumors in the sigmoid colon (arrow and arrowheads).

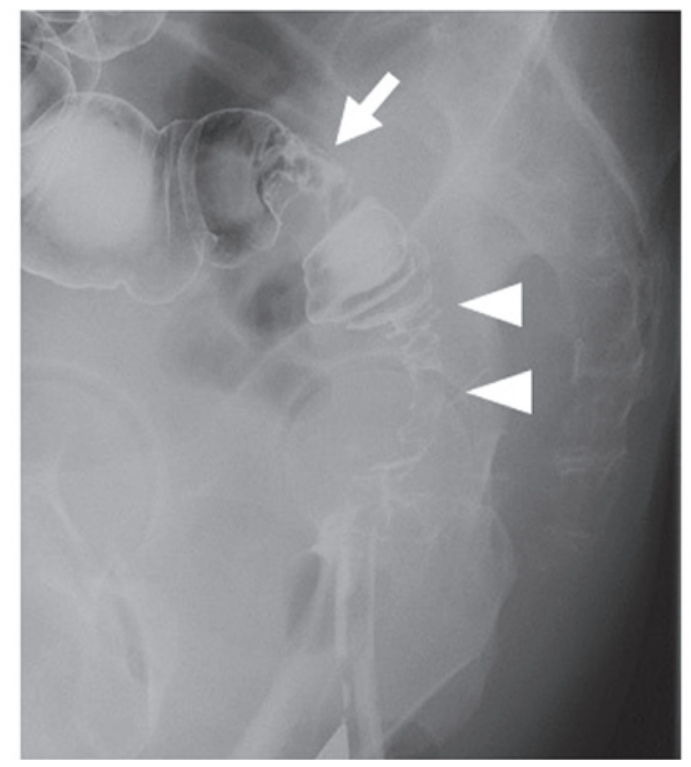

Figure 2. A water-soluble contrast enema revealed the tumor site (arrow) and the irregular stricture of the sigmoid colon between the tumor and the anastomosis site (arrowheads).

intestinal radiation damage and mucinous histology, were observed. Castro et al (5) reported that, in 12 of 26 tumors, there was no mucin production and, among the 26 cases, 4 exhibited no histological evidence of radiation damage.

Radiation proctocolitis has been reported in $5-11 \%$ of patients treated for gynecological cancers $(12,13)$. Colonic strictures, erosions and fistulae are common sequelae of radiation therapy of the gastrointestinal tract, and may result in refractory pain, tenesmus and changes in bowel habits (4). From these findings, such as the fistula of the sigmoid colon 6 years after surgery, colonic stricture and erosions, as well as the clinical symptoms, such as anal pain and frequent defecation, the suspicion of chronic radiation proctocolitis was raised in the present case.

Considering the macroscopic type, as compared to typical CRCs, radiation-associated CRCs often have a non-polypoid, diffusely infiltrating appearance rather than a polypoid or localized ulcerative appearance with a well-defined tumor margin (8). In the present case, the two flat sigmoid tumors had a diffusely infiltrating appearance, without a well-defined tumor margin. Levitt et al (7) found synchronous multiple rectal cancers within the radiation fields in 2 of 5 patients with rectal cancer following pelvic irradiation, and hypothesized that radiation exerted a 'field effect' for carcinogenesis on the 

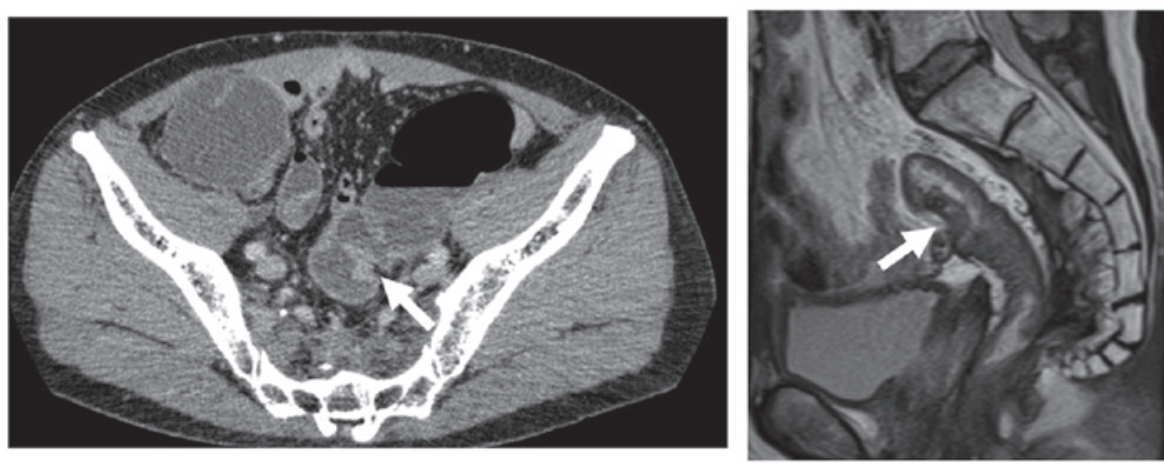

Figure 3. Contrast-enhanced computed tomography (left panel) and magnetic resonance imaging (right panel) showing the mass in the sigmoid colon (arrows).
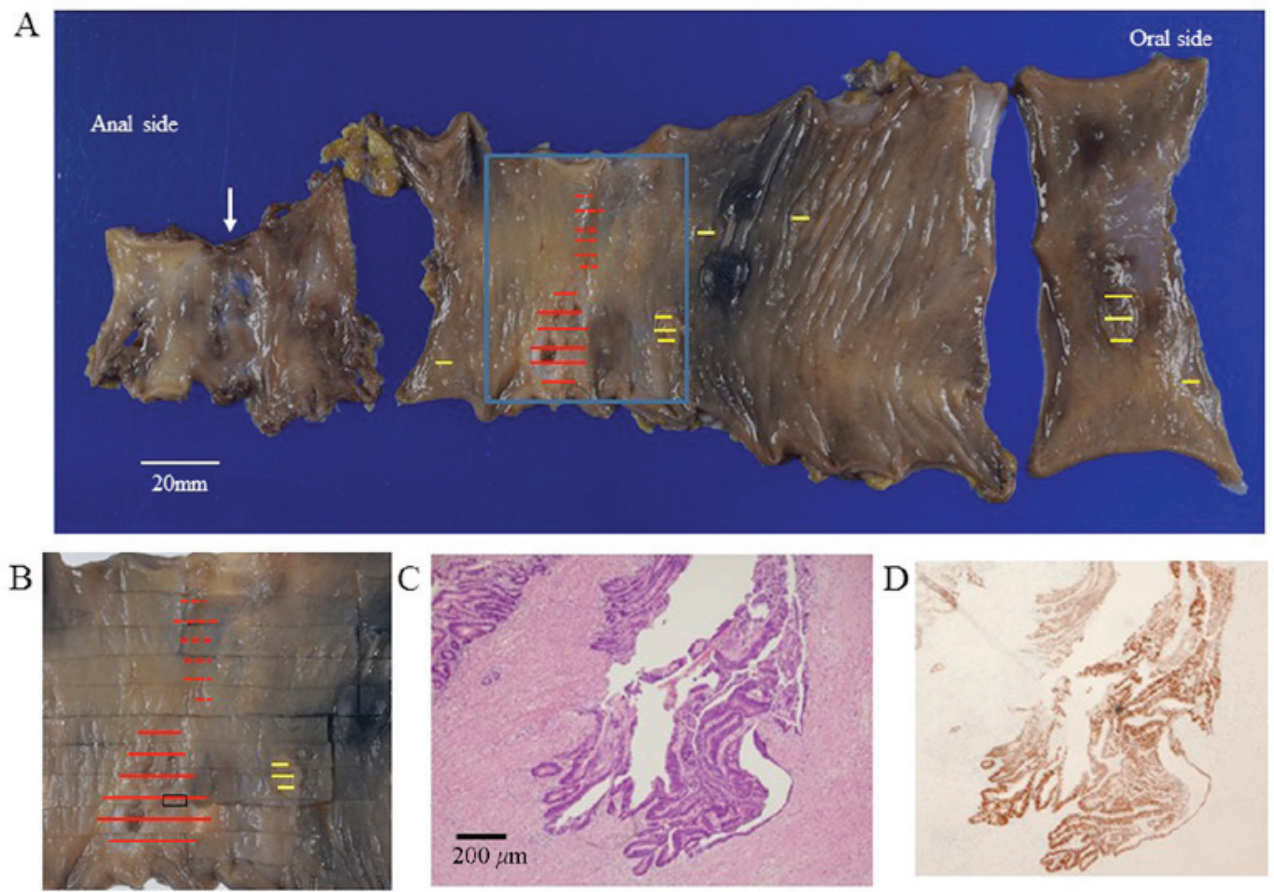

Figure 4. Macroscopic findings of the resected specimen. (A) On macroscopic examination, the resected rectum and sigmoid colon contained two separate tumors and six flat polyps (yellow lines). The first tumor, invading the subserosa, was located $8 \mathrm{~cm}$ from the distal margin (red lines), and the second tumor, invading the submucosa, was located near the first (red dotted lines). The anastomosis site was located $4 \mathrm{~cm}$ from the distal margin (arrow). (B) Magnified image of the square indicated in (A). (C) On histopathological examination, the first tumor was diagnosed as well-differentiated adenocarcinoma with invasion of the subserosa [section as indicated in (B)]. (D) On immunohistochemical examination, p53 overexpression was observed in the first tumor.

rectal mucosa. In the present case, the two flat tumors and 6 flat polyps were all observed in the resected specimens and, thus, the radiation field effect may be involved in our case. Therefore, based on the macroscopic findings and clinical characteristics of chronic radiation colitis, the two flat tumors in the present case were considered to be radiation-associated sigmoid colon cancer.

The histopathological characteristics of late radiation injury of the colorectum are characterized by severe vascular changes, such as subintimal fibrosis and hyaline sclerosis. The majority of the cases reported these findings in women with cervical cancer who received high-dose irradiation, with a dose ranging from 50 to $82 \mathrm{~Gy}(8,10,13)$. None of these vascular changes were observed in the present case; thus, we consider our case to have received lower-dose irradiation compared with previously reported cases. The dose applied in our case was 50 Gy and was focused on the rectal cancer, which had already been resected. Of note, Palmer et al reported that patients who received low-dose irradiation for benign gynecological conditions had a higher rate (3.32\%) of rectal cancer compared with those who had received high-dose irradiation (1.4\%) for cervical cancer; they suggested that the relatively low irradiation dose exerted a growth-stimulating effect that the high dose did not.

However, similar to the present case, the minority of early-onset cases may be attributed to common hereditary CRC syndromes (14). At present, two independent genetic pathways are known to be involved in colorectal carcinogenesis: One is the mutator phenotype pathway, which involves dysfunction of DNA mismatch repair machinery; tumors in this pathway frequently exhibit MSI (15). The other pathway is multistep carcinogenesis, in which accumulation of mutations 
in the adenomatous polyposis coli, KRAS, p53 and DPC genes contributes to tumorigenesis (16). In the present case, there was no detected hereditary tendency for malignancy, and there was no MSI, although the first flat tumor harbored a p53 mutation. Similar to the present case, Minami et al (17) reported that p53 protein overexpression was detected using immunohistochemistry in 7 of 9 patients with radiation-associated colon cancer; however, they found no definite correlation between the overexpression of p53 in radiation-associated and spontaneous colon cancers. This suggested that the occurrence of p53 gene mutations was not specific to the carcinogenesis of radiation-associated colon cancers compared with spontaneous colon cancers.

In the present case, the interval from radiation treatment to diagnosis of sigmoid colon cancer was 19 years. In the largest published series, the peak incidence of post-irradiation CRC was between 5 and 10 years after the completion of radiotherapy, while 1 carcinoma was diagnosed 45 years after irradiation (5). However, the effect of the radiation dose on the interval period has not been investigated. It is therefore possible that higher doses of irradiation may be associated with an earlier incidence of CRC (7). From these findings, the increased risk of CRC may start at 5 years and persist for $>40$ years after irradiation.

Clinically, the presenting symptoms of abnormal bowel movement, diarrhea and dyschezia are similar between radiation proctocolitis and radiation-associated colon cancer. Additionally, it is difficult to detect curable CRC using laboratory parameters. These factors may account for the delayed diagnosis and advanced stage of colon cancer at the time of detection $(18,19)$. In the present case, colonoscopy was performed to diagnose the cause of the anal pain, and sigmoid colon cancer was detected incidentally. Therefore, there is a need for earlier detection of CRC that develops in the setting of proctocolitis following irradiation. Patients with radiation colitis involving frequent diarrhea, dyschezia and tenesmus, similar to the present case, require long-term surveillance to prevent the growth of radiation-associated cancer.

We herein present a case of a radiation-associated colon cancer in a patient who had undergone low anterior resection for rectal cancer following preoperative radiotherapy 19 years prior. The increased risk of CRC may start at 5 years and persist for $>40$ years after irradiation. For cases of chronic proctocolitis, such as colonic stricture, fistulae and abnormal bowel movement, long-term surveillance with a colonoscope should be considered after irradiation in patients undergoing previous pelvic radiotherapy.

\section{References}

1. Wang J, Boerma M, Fu Q and Hauer-Jensen M: Significance of endothelial dysfunction in the pathogenesis of early and delayed radiation enteropathy. World J Gastroenterol 13: 3047-3055, 2007.
2. Andreyev HJ: Gastrointestinal problems after pelvic radiotherapy: The past, the present and the future. Clin Oncol (R Coll Radiol) 19: 790-799, 2007.

3. Birgisson H, Påhlman L, Gunnarsson U and Glimelius B: Occurrence of second cancers in patients treated with radiotherapy for rectal cancer. J Clin Oncol 23: 6126-6131, 2005.

4. Ashburn JH and Kalady MF: Radiation-induced problems in colorectal surgery. Clin Colon Rectal Surg 29: 85-91, 2016.

5. Castro EB, Rosen PP and Quan SH: Carcinoma of large intestine in patients irradiated for carcinoma of cervix and uterus. Cancer 31: 45-52, 1973.

6. Jao SW, Beart RW Jr, Reiman HM, Gunderson LL and Ilstrup DM: Colon and anorectal cancer after pelvic irradiation. Dis Colon Rectum 30: 953-958, 1987.

7. Levitt MD, Millar DM and Stewart JO: Rectal cancer after pelvic irradiation. J R Soc Med 83: 152-154, 1990.

8. Tamai O, Nozato E, Miyazato H, Isa T, Hiroyasu S, Shiraishi M, Kusano T, Muto Y and Higashi M: Radiation-associated rectal cancer: Report of four cases. Dig Surg 16: 238-243, 1999.

9. Shadad AK, Sullivan FJ, Martin JD and Egan LJ: Gastrointestinal radiation injury: Symptoms, risk factors and mechanisms. World J Gastroenterol 19: 185-198, 2013.

10. Tsuji T, Sawai T, Nakagoe T, Hidaka S, Shibasaki S, Tanaka K, Nanashima A, Yamaguchi H, Yasutake T and Tagawa Y: Genetic analysis of radiation-associated rectal cancer. J Gastroenterol 38: 1185-1188, 2003

11. Hotchi M, Shimada M, Kurita N, Iwata T, Sato H, Morimoto S, Yoshikawa K, Higashijima J, Miyatani T, Mikami C and Kashihara H: Short-term results of laparoscopic surgery after preoperative chemoradiation for clinically staged T3 and T4 rectal cancer. Asian J Endosc Surg 5: 157-163, 2012.

12. Perez CA, Breaux S, Bedwinek JM, Madoc-Jones H, Camel HM, Purdy JA and Walz BJ: Radiation therapy alone in the treatment of carcinoma of the uterine cervix. II. Analysis of complications. Cancer 54: 235-246, 1984.

13. Tomori H, Yasuda T, Shiraishi M, Isa T, Muto Y and Egawa H: Radiation-associated ischemic coloproctitis: Report of two cases. Surg Today 29: 1088-1092, 1999.

14. Yurgelun MB, Masciari S, Joshi VA, Mercado RC, Lindor NM, Gallinger S, Hopper JL, Jenkins MA, Buchanan DD, Newcomb PA, et al: Germline TP53 mutations in patients with early-onset colorectal cancer in the colon cancer family registry. JAMA Oncol 1: 214-221, 2015.

15. Malkhosyan S, Rampino N, Yamamoto $\mathrm{H}$ and Perucho M: Frameshift mutator mutations. Nature 382: 499-500, 1996.

16. Fearon ER and Vogelstein B: A genetic model for colorectal tumorigenesis. Cell 61: 759-767, 1990.

17. Minami K, Matsuzaki S, Hayashi N, Mokarim A, Ito M and Sekine I: Immunohistochemical study of p53 overexpression in radiation-induced colon cancers. J Radiat Res 39: 1-10, 1998.

18. Shirouzu K, Isomoto H, Morodomi T, Ogata Y, Araki Y and Kakegawa T: Clinicopathologic characteristics of large bowel cancer developing after radiotherapy for uterine cervical cancer. Dis Colon Rectum 37: 1245-1249, 1994.

19. Arai T, Nakano T, Fukuhisa K, Kasamatsu T, Tsunematsu R, Masubuchi K, Yamauchi K, Hamada T, Fukuda T, Noguchi $\mathrm{H}$, et al: Second cancer after radiation therapy for cancer of the uterine cervix. Cancer 67: 398-405, 1991. 